\title{
Wakefields in a cluster plasma
}

\author{
M. W. Mayrø, ${ }^{1,}{ }^{*}$ L. Ceurvorst, ${ }^{1, \dagger}$ M. F. Kasim, ${ }^{1}$ J. D. Sadler, ${ }^{1, \$}$ B. Spiers,${ }^{1}$ K. Glize, ${ }^{2}$ A. F. Savin, ${ }^{1}$ \\ N. Bourgeois, ${ }^{2}$ F. Keeble, ${ }^{3}$ A. J. Ross, ${ }^{1}$ D. R. Symes, ${ }^{2}$ R. Aboushelbaya, ${ }^{1}$ R. A. Fonseca,,${ }^{5,6}$ \\ J. Holloway, ${ }^{1}$ N. Ratan, ${ }^{1}$ R. M. G. M. Trines, ${ }^{2}$ R. H. W. Wang, ${ }^{1}$ R. Bingham, ${ }^{2,4}$ L. O. Silva, ${ }^{6}$ \\ P. N. Burrows, ${ }^{1}$ M. Wing, ${ }^{3}$ P. P. Rajeev, ${ }^{2}$ and P. A. Norreys ${ }^{1,2}$ \\ ${ }^{1}$ Department of Physics, University of Oxford, Oxford OX1 3PU, United Kingdom \\ ${ }^{2}$ Central Laser Facility, STFC, Rutherford Appleton Laboratory, Didcot, OX11 OQX, United Kingdom \\ ${ }^{3}$ Department of Physics and Astronomy, University College London, Gower Street, \\ London WC1E 6BT, United Kingdom \\ ${ }^{4}$ Department of Physics, University of Strathclyde, Glasgow, G4 ONG, United Kingdom \\ ${ }^{5}$ ISCTE - Instituto Universitário de Lisboa, 1649-026 Lisbon, Portugal \\ ${ }^{6}$ GoLP/Instituto de Plasmas e Fusão Nuclear, Instituto Superior Tecnico, \\ Universidade de Lisboa, 1049-001 Lisbon, Portugal
}

(Received 22 November 2018; revised manuscript received 13 February 2019; published 15 November 2019)

\begin{abstract}
We report the first comprehensive study of large amplitude Langmuir waves in a plasma of nanometerscale clusters. Using an oblique angle single-shot frequency domain holography diagnostic, the shape of these wakefields is captured for the first time. The wavefronts are observed to curve backwards, in contrast to the forwards curvature of wakefields in uniform plasma. Due to the expansion of the clusters, the first wakefield period is longer than those trailing it. The features of the data are well described by fully relativistic two-dimensional particle-in-cell simulations and by a quasianalytic solution for a onedimensional, nonlinear wakefield in a cluster plasma.
\end{abstract}

DOI: 10.1103/PhysRevAccelBeams.22.113501

\section{INTRODUCTION}

The frequency of electrons oscillating in a uniform plasma was first derived by Langmuir and Tonks in 1929 [1] and the behavior of Langmuir waves (also known as electron plasma waves) has been extensively studied [2-6]. Two widely studied applications of these waves are the beam-driven [7-13] and laser-driven [14-16] plasma accelerator, the latter using a high-power laser pulse propagating through a plasma to create longitudinal electric fields of up to $100 \mathrm{GV} \mathrm{m}^{-1}$ that accelerate charged particles.

The duration of the laser pulse is typically shorter than the period associated with the plasma wavelength, $\lambda_{\mathrm{p}}=2 \pi v_{\mathrm{p}} / \omega_{\mathrm{p}}$, where $v_{p} \approx c$ is the phase velocity of the plasma wave, and $\omega_{p}=\left(n e^{2} / m_{\mathrm{e}} \epsilon_{0}\right)^{1 / 2}$ is the plasma frequency (here, $n$ is the electron number density, $e$ is

\footnotetext{
"marko.mayr@physics.ox.ac.uk

Present address: Université de Bordeaux-CNRS-CEA, Centre Lasers Intenses et Applications, UMR 5107, 33405 Talence, France.

Present address: Los Alamos National Laboratory, P.O. Box 1663, Los Alamos, New Mexico 87545, USA.

Published by the American Physical Society under the terms of the Creative Commons Attribution 4.0 International license. Further distribution of this work must maintain attribution to the author(s) and the published article's title, journal citation, and DOI.
}

the elementary charge, $m_{e}$ is the electron mass, and $\epsilon_{0}$ is the permittivity of free space). Fulfilling this condition, an efficient acceleration regime, the regime, was found computationally [17]. This was followed by the generation of well-populated, high-quality beams of electrons [18-20] and subsequently the generation of quasimonoenergetic $\mathrm{GeV}$ electron bunches [21]. Since then, many experiments have been conducted pushing the frontiers of laser wakefield acceleration to higher energies and better quality beams [22-27]. Applications of accelerated particle beams are ubiquitous in the natural sciences and medicine [28-30].

Wakefields can also be used to accelerate electrons in a nonuniform, clustered plasma using gases such as argon [31-33] or methane [34]. A plasma cluster is an accumulation of ionized atoms which were formerly held together by van der Waals forces. The electrons are attracted to the positively charged ion clusters, changing the optical properties both locally and globally within the plasma. A laser propagating through a medium containing clusters is able to access the "cluster mode" which allows it to propagate in a plasma of critically overdense clusters at group velocities higher than in a uniform, nonclustered plasma [35] of same average density. The evolution of clusters irradiated by an intense laser pulse and the dependence of the laser pulse propagation on the expansion of the clusters have been investigated [31,36-42]. 
In this article, experimental data obtained from a single-shot, oblique-angle frequency-domain holography diagnostic [43] is presented, showing for the first time how the shape and wavelength of a wakefield are changed in the presence of clusters. These findings are supported by $2 \mathrm{D}$ particle-in-cell simulations which show that the wakefield wavelength decreases with increasing distance behind the drive pulse, in agreement with the experimental data. Controlling the plasma wavelength could potentially help to increase the dephasing length [15], which is one of the major limiting factors of wakefield accelerators. It is also observed for the first time that the wavefronts are curved backwards, in contrast to those in a uniform plasma. This article is structured as follows. Section II presents experimental observations from our single-shot frequency-domain holography diagnostic. Section III presents results of a one-dimensional model of nonlinear wakefields in a plasma with strong density perturbations. Section IV presents the results of two-dimensional moving-window particle-in-cell simulations supporting the observations made in the previous sections. This is followed by a discussion.

\section{EXPERIMENTAL DATA}

The experimental investigation of wakefields in a cluster plasma was conducted at the ASTRA high-power laser facility [44]; a schematic of the experiment is shown in Fig. 1. In this experiment a plasma wakefield is generated using a laser pulse with a laser wavelength of $800 \mathrm{~nm}$, energy of $480 \mathrm{~mJ}$, FWHM duration of $45 \mathrm{fs}$, spot size diameter of $29 \mu \mathrm{m}$ (FWHM) and peak intensity of $1.1 \times 10^{18} \mathrm{~W} \mathrm{~cm}^{-2}( \pm 15 \%)$ which gives a normalized laser amplitude, $a_{0}$, of 0.72 where $a_{0} \equiv 8.55 \times 10^{-10}$ $\left(I \lambda^{2}\left[\mathrm{~W} \mathrm{~cm}^{-2} \mu \mathrm{m}^{2}\right]\right)^{1 / 2}$ (here, $I$ is the intensity in $\mathrm{W} \mathrm{cm}^{-2}$ and $\lambda$ is the laser wavelength in $\mu \mathrm{m}$ ). The cluster plasma was created in a $3 \mathrm{~mm}$ wide gas jet which ejected methane with a backing pressure of 7-14 bar into vacuum. At these backing pressures, the conditions for clusterization of methane $[45,46]$ are fulfilled. The electron density was measured using a 550 fs interferometry probe beam aligned perpendicular to the driving pulse. A 10 ps delay was set between the driving and interferometer pulses to allow the electrons to homogenize.

In order to measure the shape of the wakefield a frequency-domain holography diagnostic was used [43], for which two positively chirped $400 \mathrm{~nm}, 1 \mathrm{~mJ}, 550 \mathrm{fs}$ pulses (probe and reference) propagate at an oblique angle of $8^{\circ}$ with respect to the drive pulse (Fig. 1). The reference pulse was $1.5 \mathrm{ps}$ ahead of the probe and also ahead of the ionization front, thus avoiding any phase shift. The probe and reference pulses were magnified by a factor of $55( \pm 10 \%)$, imaged at the focal plane and measured using a spectrometer [raw data is shown in Fig. 2(a)]. From this, the phase shift imprinted onto the probe pulse by the

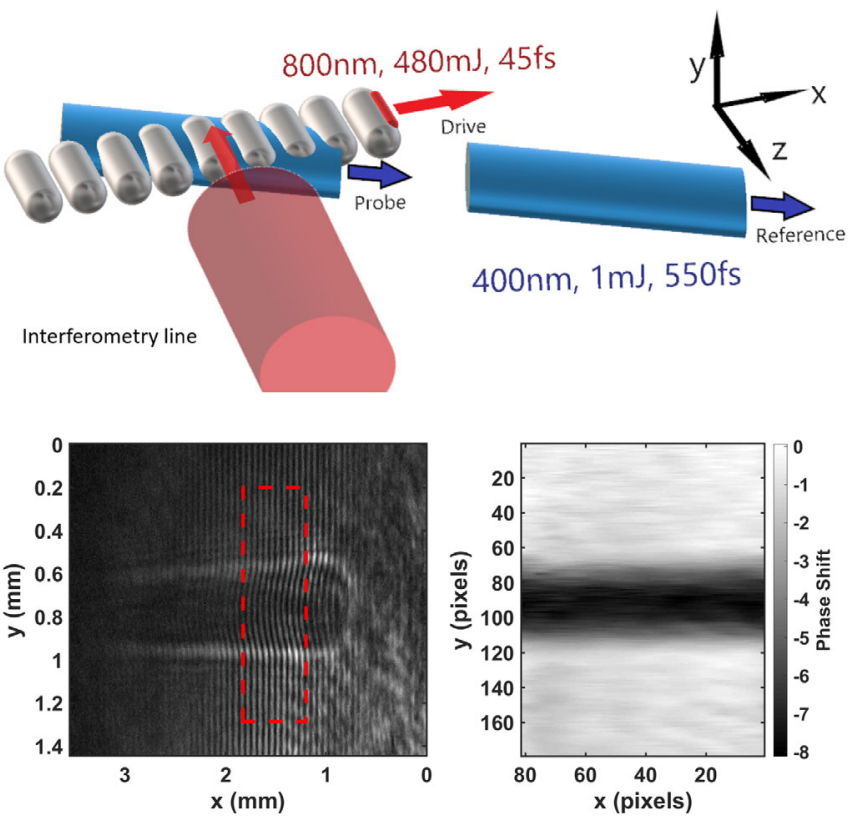

FIG. 1. Schematic of the experimental set-up, including a single-shot oblique-angle frequency domain holography diagnostic and a transverse optical probe. The drive laser pulse (red) is incident from left to right and excites a large amplitude Langmuir wave (grey). The density variations imprint a phase shift onto the probe pulse (blue) which crosses at an angle of $8^{\circ}$. The lower panels show results from the transverse interferometry measurement. The left panel shows the ionization front of the propagating laser and the fringes from which the phase shift is calculated. The right panel shows the phase shift obtained from analysing the area indicated by the red rectangle in the left panel. The plasma density was obtained from this phase shift.

density perturbations within the wakefield was recovered. Due to the linear frequency chirp of the probe pulse, the measured laser wavelength directly maps to the delay behind the drive pulse. This mapping has been applied to the axes in Figs. 2(a) and 2(b). Interference of the reference and probe pulses leads to fringes in the combined spectrum. This setup is similar to a frequencydomain streak camera [47] but measures the phase across the $x-y$-plane (using the axes defined in Fig. 1) instead of the $\mathrm{x}$-z-plane of the probe pulse. Hence, it gives a snapshot of the wakefield rather than the temporal evolution of the structure.

The phase shift was obtained using a method ([48,49]) based on a reconstruction algorithm which incorporates data from two spectrograms [Figs. 2(a1) and 2(a2)]. The Fourier transform was taken and the peak corresponding to the fringes in the spectrogram isolated. The results of a shot in which gas was used [Fig. 2(a1)] are then divided by the results of a reference shot in which no gas was used [Fig. 2(a2)]. In the latter case the reference pulse obtained no phase shift which makes it possible to reduce phase shift signals that are caused by the diagnostic rather than the laser-plasma interaction and to prevent errors arising 

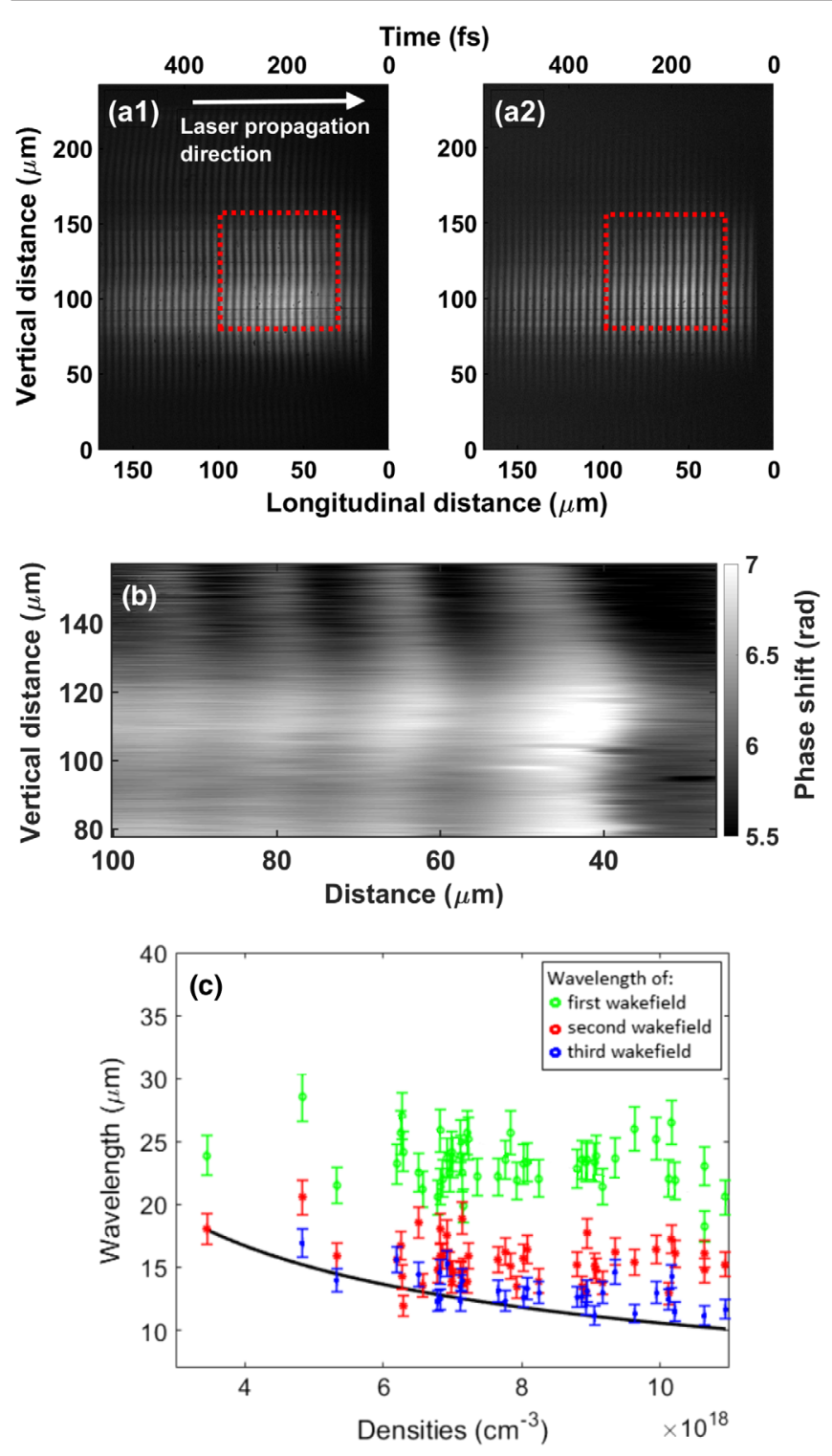

FIG. 2. (a) Spectrum of the image of the wakefield formed by the probe and reference pulses from which the phase shift across the $x-y$-plane was recovered. The drive pulse propagates to the right. The $\mathrm{x}$-axis was mapped from laser wavelength to time (upper $\mathrm{x}$-axis) using calibration data. This was multiplied by the approximated group velocity of the laser to convert to space (lower $\mathrm{x}$-axis). The $\mathrm{y}$-axis has an estimated systematic error of $\pm 10 \%$ due to the uncertainty of the magnification. (b) Processed data showing the unwrapped phase indicating the structure of the wakefield in a cluster plasma. Close-up of the area indicated by the red dashed rectangle in (a). (c) Measurement of the first (green), second (red), and third (blue) wakefield wavelengths as a function of the average plasma electron density measured by the transverse interferometry. The plot shows data from 30 separate laser shots. The black line shows the theoretical plasma wavelength for a uniform plasma. The data points were measured on-axis as the distance between successive minimum phase shifts. The error bars were obtained from the measurements for the calibration of the conversion parameter between pixel on CCD and time, $b=(1.81 \pm 0.12) \mathrm{pix} / \mathrm{fs}$. from manually shifting the peak in the analysis. The obtained results in Fig. 2(b) show a wakefield structure that differs from that typically seen in a uniform plasma case as the wavefronts are curved backwards with respect to the direction of laser propagation-in a uniform plasma, the wakefield's wavefront curves forwards. Figure 2(c) shows the plasma wavelengths of the wakefield measured by the frequency domain holography diagnostic as a function of the average plasma density. The wavelength of each wake was measured on-axis as the distance between successive phase shift minima. The observation of an increased first and second plasma wavelength (with respect to the theoretically predicted uniform plasma value) was reproducible over a range of averaged plasma density. The third wakefield wavelength coincides with the theoretical value $2 \pi c / \omega_{\mathrm{p}}$ where $\omega_{\mathrm{p}}$ is calculated using the average electron density. Whereas the third wakefield wavelength seems to scale with density as expected, the first wakefield wavelength does not seem to depend on this parameter in the same way.

\section{QUASIANALYTIC 1D MODEL}

Nonlinear, quasianalytic models for wakefield creation in one dimension $[50,51]$ use the quasistatic approximation $[52,53] \partial / \partial \tau=0$ assuming that quantities change slowly in the comoving frame of reference. In a cluster plasma, the quasi static approximation is no longer valid as the density may fluctuate strongly with respect to the co-moving variables $\xi=x-v_{g} t$ (spatial coordinate moving at the laser group velocity $v_{g}$ ) and $\tau=t$ (time). Instead of obtaining an analytical expression from integrating the continuity equation $\partial n / \partial \tau=0=\partial / \partial \xi\left[n\left(v_{g}-v_{x}\right)\right]$, one can proceed by numerically solving the coupled system of Ampere's law $\partial E / \partial t=-j_{x} / \epsilon_{0}$, Poisson equation $\partial E / \partial x=-\rho / \epsilon_{0}$ and the equation of motion in the copropagating frame of reference $\partial / \partial \tau\left(\gamma v_{x} / c\right)=\partial /$ $\partial \xi\left[\tilde{\Phi}-\gamma\left(1-\left(v_{g} v_{x}\right) / c^{2}\right)\right]$. Here, $n$ is the electron density, $v_{x}$ is the longitudinal electron velocity, $j_{x}$ is the corresponding current, $E$ is the electric field, $\epsilon_{0}$ is the vacuum permittivity, $\rho$ is the charge density, $\gamma$ is the Lorentz factor, $c$ is the speed of light in vacuum, and $\tilde{\Phi}$ is the normalized electrostatic potential. Combining Ampere's law and the Poisson equation yields

$$
\frac{\epsilon_{0}}{e n_{0} c} \frac{\partial E}{\partial t}=\left(\frac{n_{i}}{n_{0}}-\frac{\epsilon_{0}}{e n_{0}} \frac{\partial E}{\partial x}\right) \beta_{x},
$$

where $n_{i}$ is the ion density, and $\beta_{x}$ is the normalized velocity $v_{x} / c$. Changing frame of reference yields

$$
\frac{1}{c} \frac{\epsilon_{0}}{e n_{0}} \frac{\partial}{\partial \tau} E=\left(\frac{n_{i}}{n_{0}}-\frac{\epsilon_{0}}{e n_{0}} \frac{\partial E}{\partial \xi}\right) \beta_{x}+\frac{\epsilon_{0}}{e n_{0}} \frac{\partial}{\partial \xi}\left(\beta_{g} E\right) .
$$

Normalizing the equations in the comoving frame, one finds 


$$
\frac{\partial}{\partial \tilde{\tau}} \tilde{E}=\left(\frac{n_{i}}{n_{0}}-\frac{\partial \tilde{E}}{\partial \tilde{\xi}}\right) \beta_{x}+\frac{\partial}{\partial \tilde{\xi}}\left(\beta_{g} \tilde{E}\right)
$$

as well as

$$
\frac{\partial}{\partial \tilde{\tau}}\left(\tilde{p}_{x}\right)=-\tilde{E}-\frac{\partial}{\partial \tilde{\xi}}\left(\gamma-\tilde{p}_{x} \beta_{g}\right)
$$

where $\tilde{E}=e E /\left(m c \omega_{p}\right)=\omega_{p} \epsilon_{0} E /\left(e n_{0} c\right), \quad \tilde{p}_{x}=p_{x} / m c$, $\partial / \partial \tilde{\tau}=1 / \omega_{p} \partial / \partial \tau$, and $\partial / \partial \tilde{\xi}=c / \omega_{p} \partial / \partial \xi$. Using a weighted essentially nonoscillatory (WENO) scheme [54], the system of coupled partial differential equations [Eqs. (3) and (4)] is solved numerically. A WENO scheme is able to deal with sharp gradients and is, hence, more suitable for the treatment of a cluster plasma than typical PDE solver routines. As this is a fluid model, it is not possible to treat a case in which the background density $n_{0}=0$ (i.e., clusters in vacuum) so a low background density $\left(n_{0}=0.001 \times n_{\text {crit }}\right)$ was used into which the highdensity clusters $\left(n_{c l}=1.5 \times n_{\text {crit }}\right)$ are injected. To model the effect of cluster expansion in the longitundinal direction, the electron distribution was analyzed from 2D PIC simulations (see Sec. IV) for different cluster parameters. The simulations show that the cluster expansion scales with the maximum cluster density. This is expected since the Coulomb expansion force $F_{c} \propto n_{e} /$ $n_{c l} \times r / 2$ scales with electron density which is applicable if large amounts of charge are displaced at the same time by the Lorentz force. This will be further discussed in Sec. IV.

Figure 3 shows the one-dimensional results of the WENO scheme of both uniform (upper panel) and cluster (lower panel) plasma. This quasianalytic model shows that wakefields formed in a plasma of (artificially) expanding clusters have a longer wavelength at the front which then shortens toward the uniform plasma limits when the clusters have expanded more strongly. The plasma wavelength of $25.3 \mu \mathrm{m}$ corresponding to the background density $n_{0}$ is normalized to $2 \pi c / \omega_{p}$ in the upper panel and, hence, equals 1 as expected. The uniform result which acts as a reference reproduces known results of nonlinear wakefields well $[50,51]$. The lower panel is normalized to the same plasma wavelength as the upper panel. However, the average density is $\left\langle n_{e}\right\rangle=0.0081 \times$ $n_{\text {crit }}$ which would lead to a plasma wavelength of $8.9 \mu \mathrm{m}$ in a uniform plasma. The measured wavelengths in the simulation obtained from filtering the longitudinal electric field using a Gaussian smoothing kernel with standard deviation of 250 cells (corresponding to $2.21 \mu \mathrm{m}-\mathrm{a}$ distance on the order of the cluster separation) are $19.7 \mu \mathrm{m}, 10.1 \mu \mathrm{m}, 8.6 \mu \mathrm{m}$ and $8.5 \mu \mathrm{m}$. The fact that the last two wavelengths are slightly shorter than the theoretical, uniform value of $8.9 \mu \mathrm{m}$ may be due to the filtering process. In the uniform plasma case the electron density coincides with the charge density. In the case of
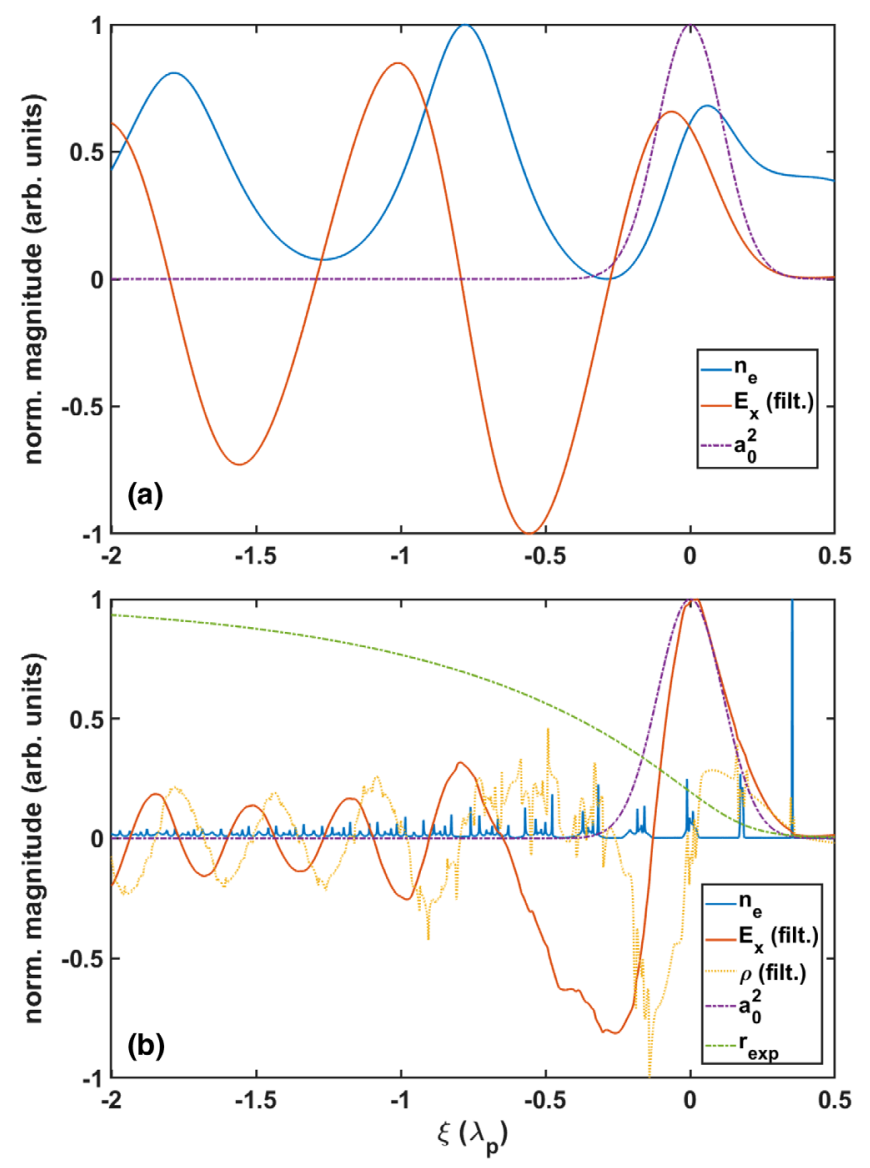

FIG. 3. Results of the 1D model of a wakefield in a co-moving frame showing the difference in wavelength of the smoothed longitudinal electric field $E_{x}$ for two different cases. The top panel shows a reference solution for a wakefield in a uniform plasma of background density $n_{0}=0.001 \times n_{\text {crit }}$ and the $\xi$-axis is normalized to $\omega_{p}\left(n_{0}\right) / c$. The bottom panel shows a plasma of background density $n_{0}=0.001 \times n_{\text {crit }}$, in which clusters of density $1.5 \times n_{\text {crit }}$ are injected from the right boundary. They artificially expand according to the expansion function $r_{\text {exp }}$. The average density is $n_{a v}=0.0081 \times n_{\text {crit }}$ leading to a predicted plasma wavelength of $8.9 \mu \mathrm{m}$. The electric field was smoothed using a Gaussian smoothing kernel.

a strongly fluctuating electron density in the vicinity of sharp ion density perturbations the electron density may differ strongly from the charge density. Therefore, the wakefield cannot immediately be seen in a plot of the electron density. The coupled equations were solved on a grid of 10000 cells. The shape of the cluster was a super-Gaussian of form $\exp \left(-(x / r)^{6}\right)$ rather than a step function to avoid instabilities and the associated FWHM cluster radius $r$ was $70.8 \mathrm{~nm}$. Both the average density and the cluster radius were chosen to be higher than the predicted experimental values since the nature of the one dimensional geometry makes it necessary to occupy the space with more clusters per wavelength in order to have a relevant impact on the uniform background density. 


\section{2D PARTICLE-IN-CELL SIMULATIONS}

In order to verify the observations from the obliqueangle frequency-domain holography diagnostic, we conducted 2D particle-in-cell moving-window simulations using the fully relativistic code OSIRIS [55]. Estimates for the cluster radius and cluster peak density were taken from the literature [56]. The empirically derived Hagena parameter describing the abundance of clusters at given gas and nozzle parameters was evaluated for typical conditions in [57].

Converting from dimensionless OSIRIS units to physical units using a fundamental laser wavelength of $800 \mathrm{~nm}$, the clusters initialized at the boundary had a circular shape, radius of $\sim 12.7 \mathrm{~nm}$, electron and ion temperatures of $10 \mathrm{eV}$ and densities of $1.5 n_{\text {crit }}=2.61 \times 10^{21} \mathrm{~cm}^{-3}$ where $n_{\text {crit }}=$ $\omega_{0}^{2} m_{e} \epsilon_{0} / e^{2}$ is the critical density of plasma for the drive pulse frequency $\omega_{0}$. The averaged electron density in the simulation box was $0.004 n_{\text {crit }}$, corresponding to $6.97 \times$ $10^{18} \mathrm{~cm}^{-3}$ which lies within the range of plasma density measured in the experiment. The normalized laser amplitude $a_{0}$ was set to be 0.72 and the laser had linear p-polarization with a Gaussian (longitudinal and transverse) shape. The FWHM duration of the pulse was varied and the transverse FWHM was kept constant at $32 \mu \mathrm{m}$. For this simulation, 160 cells per laser wavelength (corresponding to $14000 \times 12000$ cells) were used, and there were $16 \times 16$ electron macro-particles per cell and $4 \times 4$ ion macroparticles per cell. The particle boundaries were absorbing and the longitudinal (transverse) field boundaries were of type "open" ("Lindman" [58]). The simulation time step was 16.6 as and all figures shown were taken at the last simulation time step corresponding to $0.42 \mathrm{ps}$ of propagation through the plasma.

Figure 4 shows the results obtained from the 2D, moving-window simulations. Two equivalent simulations using a laser duration of 45 fs are compared, one using a cluster plasma [panels (a), (b), (c), and (e)] and one using a uniform plasma distribution [panels (d) and (f)] of the same average density. In the top panel, the initially over-dense clusters (injected from the right-hand boundary of the simulation box) start expanding as soon as the laser encounters them. Detailed analysis of these density patterns reveals that the electrons oscillate around the positively charged ion clusters to form dipolelike structures synchronized with the laser field (see Fig. 5). The electron motion is directly linked to the laser pulse electric field. Within one half-oscillation of field strength the majority of electrons are located on one side of the ion cluster. Due to the significant amount of time the electrons spend outside of an ion cluster and within an electron plume, the repulsive Coulomb force creates a momentum distribution which prevents some electrons from returning directly to the ion cluster. Hence, the repulsive Coulomb force between electrons outside of the cluster starts to increase the density
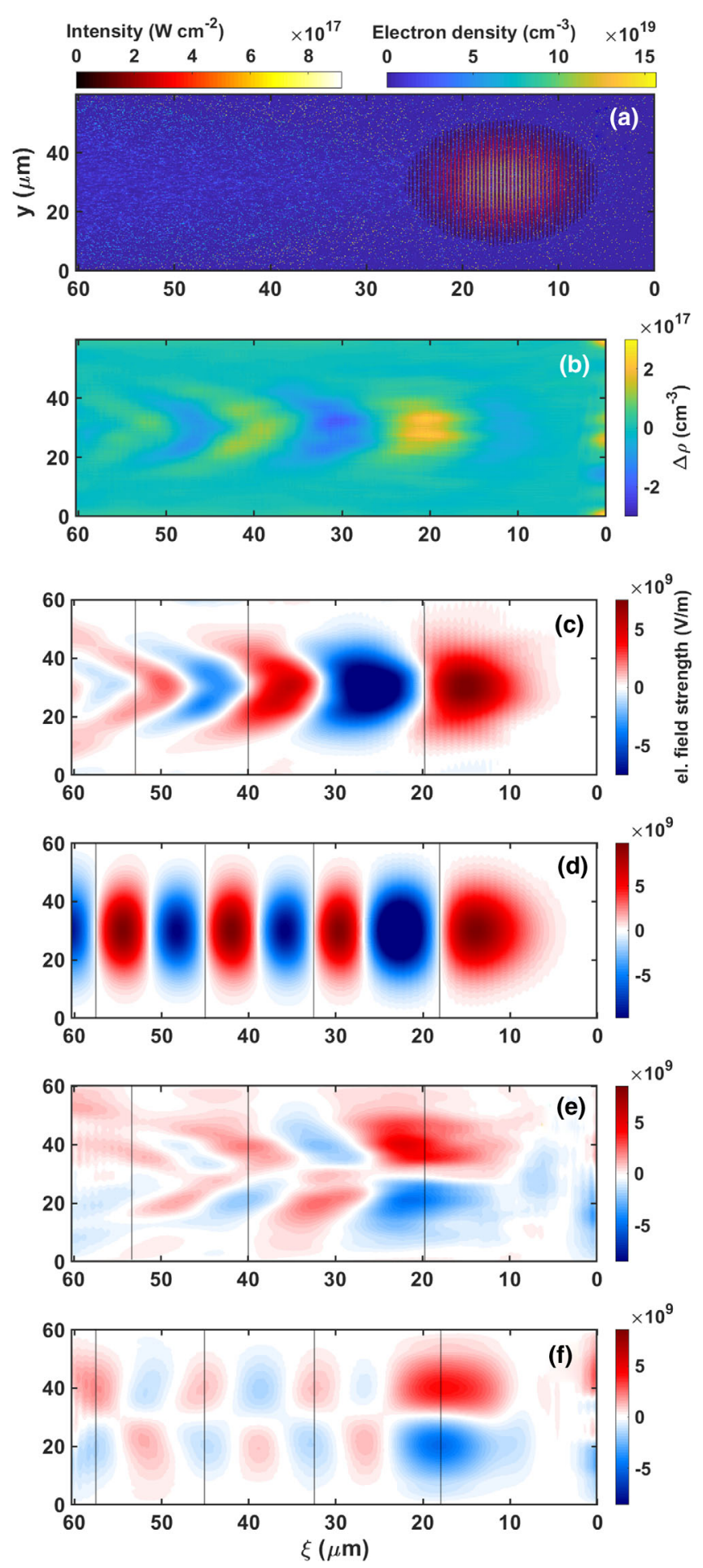

FIG. 4. Results of the 2D moving-window particle-in-cell simulations where the laser pulse propagates toward the righthand side of the window. Panel (a) shows the electron density and the laser intensity in a cluster plasma. Panel (b) shows perturbations in the charge density. Panel (c) shows the longitudinal electric field in propagation direction of the wakefield in a cluster plasma. Panel (d) shows an equivalent simulation for a uniform plasma. Panel (e) shows the transverse electric field in a cluster plasma. Panel (f) shows an equivalent simulation for a uniform plasma. The laser duration for both simulations was $45 \mathrm{fs}$. The average electron density was $6.97 \times 10^{18} \mathrm{~cm}^{-3}$. 


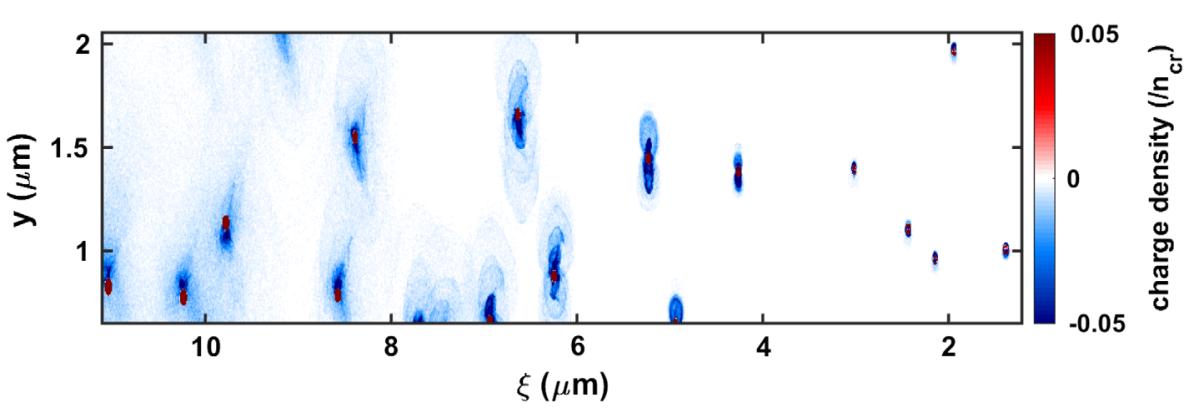

FIG. 5. Close-up of the charge density of expanding clusters from a 2D moving-window particle-in-cell simulation. Ion clusters are plotted in red, electrons in blue. The circular clusters were initialised with density of $1.5 \times n_{c r}$ and radius of $12.7 \mathrm{~nm}$. The laser pulse is centered outside of the depicted area at $17 \mu \mathrm{m}$.

of electrons that are not oscillating about the ion cluster. As an electron is expelled further away from its associated cluster, it begins to be affected by electrostatic forces attracting it to other, neighboring ion clusters. Thus, the effect of the electrostatic potential confining an electron to the vicinity of any given cluster is reduced. As this density of dissociated electrons continues to increase behind the laser pulse to the left (see Fig. 5), the plasma frequency increases accordingly. This causes the plasma wavelength to decrease until all clusters have homogenized, at which time it matches that of a uniform plasma of the same average density.

Panel (b) of Fig. 4 shows the perturbations in the charge density driven by a laser pulse in a cluster plasma and panels (c) and (d) show the longitudinal electric field for cluster and uniform plasma respectively. The results have been smoothed using a two-dimensional Gaussian smoothing kernel with standard deviation of 350 cells (corresponding to $1.75 \mu \mathrm{m}$ ) to average the quickly varying fields around the clusters. The wakefield shape shows behavior similar to that observed in the experimental data [Fig. 4(b)]. The wavefronts curve backwards and the first plasma wavelength is larger than those trailing it. In contrast to these results, the bottom panel in Fig. 4 (again, smoothed over $1.75 \mu \mathrm{m}$ ) shows that the wavefronts curve slightly in the opposite direction in a uniform plasma, as expected due to the relativistic mass increase on axis. The vertical lines marking the boundaries between complete oscillations of the plasma wave make it possible to compare the wakefield wavelengths for the different cases. The theoretical (uniform plasma) value for the Langmuir wavelength [15] in a simulation using a density of $6.97 \times 10^{18} \mathrm{~cm}^{-3}$ is calculated to be $12.6 \mu \mathrm{m}$. While the plasma wavelength in the uniform plasma case is very

(a)

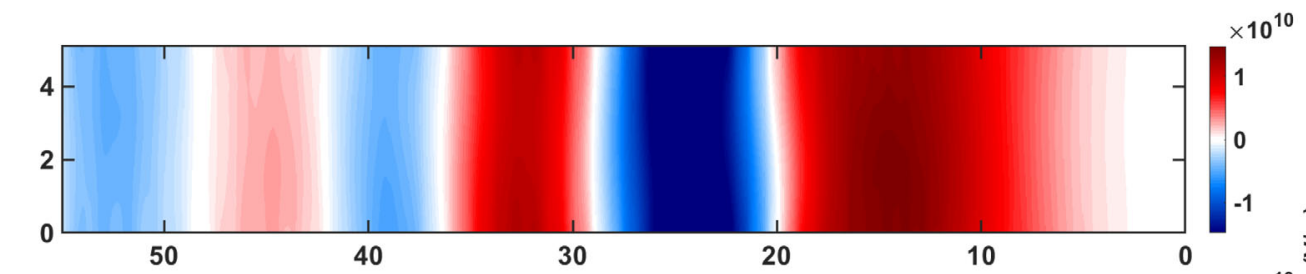

(b)

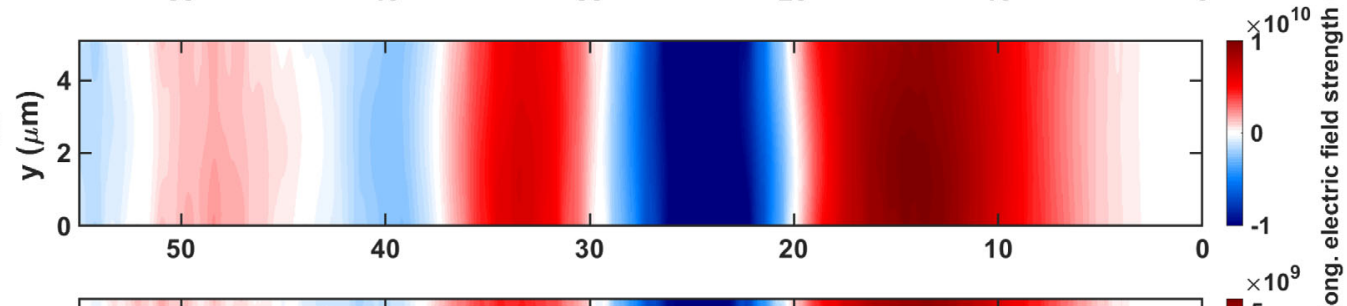

(c)

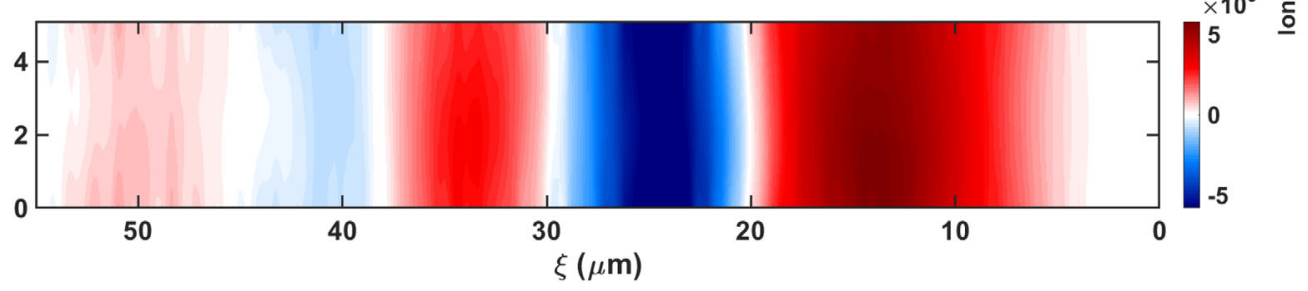

FIG. 6. Results of 2D PIC simulations for different laser amplitudes. The longitudinal electric field is plotted for (a) $a_{0}=0.9$, (b) $a_{0}=0.72$, and (c) $a_{0}=0.5$. The field is smoothed over 350 cells. The laser pulse is centered at $17 \mu \mathrm{m}$. 
(a)

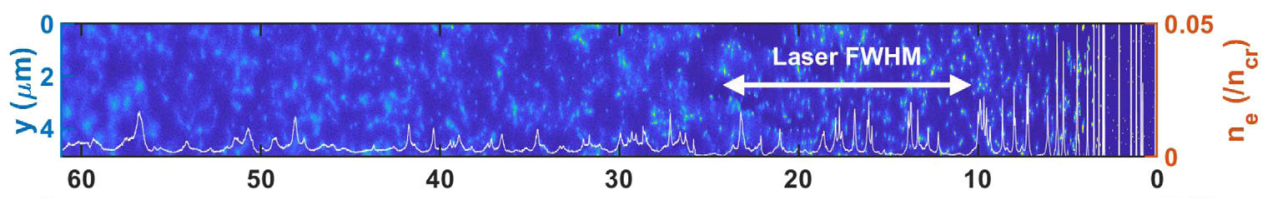

(b)

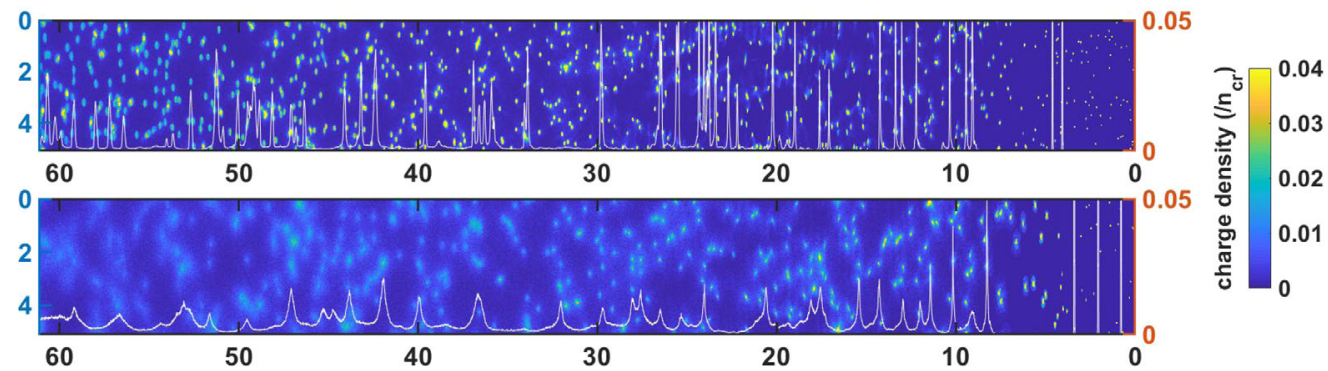

(d)

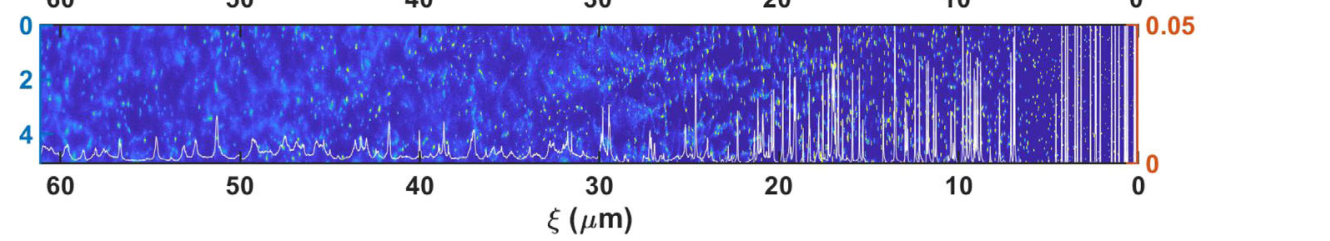

FIG. 7. Results of a 2D moving-window particle-in-cell simulation showing the electron charge density for varying initial cluster parameters. Each subplot shows the cluster expansion toward the left after the clusters encounter the plane wave laser pulse [indicated in (a)] as well as a horizontal lineout averaged over 50 cells in the center. The initial cluster parameters are (a) $n_{c l} / n_{c r}=1.5, r=6.4 \mathrm{~nm}$, (b) $n_{c l} / n_{c r}=0.7, r=12.7 \mathrm{~nm}$, (c) $n_{c l} / n_{c r}=1.5, r=12.7 \mathrm{~nm}$, and (d) $n_{c l} / n_{c r}=0.7, r=6.4 \mathrm{~nm}$. The FWHM of the laser pulse duration used in the simulation (45 fs) is indicated by the white double-arrow. The laser pulse is centered at $17 \mu \mathrm{m}$. The boundary conditions in the y-direction are chosen to be periodic. The normalized laser amplitude for all simulations was $a_{0}=0.72$.

close to the theoretical value (namely $14.9 \mu \mathrm{m}, 12.5 \mu \mathrm{m}$, $12.5 \mu \mathrm{m})$, the presence of clusters leads to an increase in plasma wavelength for the first and second wakefield structure $(20.1 \mu \mathrm{m}, 13.3 \mu \mathrm{m})$. It is worth noting that the cluster plasma wakefield wavelengths in Fig. 4 are measured to be shorter than the experimentally observed ones (approximately $23 \mu \mathrm{m}$ and $17 \mu \mathrm{m}$ ) at corresponding density. The reason for this difference between simulation and experiment may be linked to the unknown cluster conditions in the experiment.

Panels (e) and (f) show the transverse electric field, using the same smoothing kernel as before. In the simulation, the laser is polarized in the plane depicted in panels (e) and (f). Since its field strength's magnitude is greater than that of the electric fields caused by the wakefield, it had to be separated. The laser field was subtracted by taking the Fourier transform and deleting the high-frequency laser oscillations. Depicted is the inverse Fourier transform. This process is equivalent to the use of a low-pass filter with a cut-off frequency of $\omega_{c o}<\omega_{0}$.

In order to understand the curvature of the wakefield structure additional two-dimensional PIC simulations were performed with a reduced number of grid-points in the $y$-direction. In these simulations the normalized laser amplitude was varied from 0.5 to 0.9. Figure 6 shows that a laser of higher intensity leads to shorter wavelengths. For $a_{0}=0.9$ the laser periods were $16.5 \mu \mathrm{m}$ and $11.6 \mu \mathrm{m}$, for $a_{0}=0.72$ the laser periods were $17.6 \mu \mathrm{m}$ and $14.3 \mu \mathrm{m}$, and for $a_{0}=0.5$ the laser periods were $18.2 \mu \mathrm{m}$ and $16.2 \mu \mathrm{m}$. This effect can be related to the quicker cluster expansion and hence an increased free (not associated with the cluster bulk motion and subject of the cluster polarization effect) electron density behind the laser pulse. The plasma wavelength scales as $n^{-1 / 2}$. The simulation used $12000 \times 1000$ cells, a timestep of 11.9 as, $32 \times 32(2 \times 2)$ electron (ion) macro-particles per cell, a transversally plane wave with a longitudinal Gaussian profile with a FWHM duration of $45 \mathrm{fs}$. The longitudinal (transversal) boundary conditions for the electric field were of type "Lindman" (periodic). It is likely that the plasma wavelengths are shorter compared to the full width simulations due to the use of periodic boundary conditions for the electromagnetic fields.

The relation between varying laser amplitude and wakefield wavelength (or cluster expansion) can be linked to the curvature as follows. In a Gaussian laser pulse the intensity transversely drops off from the peak value on the symmetry axis. If the decrease in laser amplitude in y-direction is continuous rather than stepwise (as in Fig. 6) the increase in wavelength is also continuous resulting in a wakefield shape that is bent backwards.

In order to quantify the effect cluster parameter uncertainties have on the cluster expansion and, hence, on the wakefield creation, four further simulations were 
performed (Fig. 7) in which the cluster parameters were varied as follows:

\begin{tabular}{lcc}
\hline \multicolumn{3}{c}{ Simulation parameters } \\
\hline Simulation & Cluster radius $(\mathrm{nm})$ & Cluster \\
\hline (a) & 6.4 & 1.5 \\
(b) & 12.7 & 0.7 \\
(c) & 12.7 & 1.5 \\
(d) & 6.4 & 0.7 \\
\hline
\end{tabular}

Very different expansion patterns are observed from the simulations, indicating that the wakefield properties depend on those cluster parameters that can be tuned using different gas jet backing pressures or laser pre-pulses which would increase the cluster expansion before interaction with the drive pulse. It can be seen that for large, underdense clusters [Fig. 7(b)] the disintegration of the clusters is slower. This can be linked to the lower electron density outside of the ion cluster which leads to a smaller intracluster Coulomb force separating the electrons. Therefore, the amount of electrons involved in the wakefield creation process through the ponderomotive push is smaller. Hence, by tuning the cluster parameters one should be able to control wakefield properties such as curvature giving control over dephasing length and emittance. However, a quantitave investigation of the exact relation of this process is beyond the scope of this paper.

\section{DISCUSSION}

It has been shown theoretically using a two-dimensional calculation of the dispersion relation of electrostatic waves [59] that the abundance of clusters in a background gas leads to an expansion of the plasma wavelength and that this effect scales with the cluster radius and the clusterization fraction. The model predicts that the plasma wavelength will increase as cluster radii increase, and as a larger fraction of the plasma particles are localized in clusters. This theory supports the findings, even though it has to be pointed out that the two-dimensional particle-in-cell simulations were initialised without background gas. However, due to the quick disintegration of the overcritical clusters into low-density electron clouds it can be assumed that the described effect of cluster polarization still plays a role in increasing the plasma wavelength. Once clusters have disintegrated completely the polarization effect will cease to exist. The primary contribution to variations in plasma wavelength is attributed to the increasing "free" electron density-electrons still bound to clusters will restrict electrostatic effects to the vicinity of the cluster, so as more electrons dissociate from their clusters more truly collective behavior is able to manifest. The one-dimensional model of Sec. III shows that the contribution of an extreme density perturbation has a lower impact on the wakefield wavelength than the same charge density spread over a larger area.

The dephasing length over which electrons can be accelerated can be written as $L_{d}=\left(\lambda_{p} \times v_{g}\right) /$ $\left[2 \times\left(c-v_{g}\right)\right]$, where $v_{g}$ is the group velocity of the laser. If the length of the accelerating structure (i.e., the wakefield wavelength) can be expanded using clusters, this could, in addition to the increase of the group velocity in the cluster mode mentioned above, extend the dephasing length.

Even though no electrons were injected into the wakefield in the data presented in this paper, a qualitative estimate of the preservation of the electron beam emittance can be estimated. Keeping the emittance minimal requires that the electron beam waist needs to be smaller than the radial extent of the focusing fields. Looking at the extent of the transverse (focusing) fields depicted in Figs. 4(e) and 4(f) one can see that this holds for typical electron beam diameters in both cases. Furthermore, the transverse electric fields in the cluster plasma case and the uniform plasma case need to be of the same magnitude in order to lead to comparable emittance. This holds in the regime we are investigating. In addition, there has to be a region in which accelerating (longitudinal fields) overlap with focusing (transverse) fields. As can be seen from Fig. 4 this region exists for both cases, in uniform and cluster plasma. However, this focusing and accelerating bucket is getting smaller in subsequent wakes in the cluster case. Hence, we predict a change in emittance for injected electrons between different buckets. While the emittance should be preserved in both cases for the first wakes, the emittance of trapped electrons in later wakes will worsen in the cluster case.

\section{CONCLUSION AND OUTLOOK}

In summary, the first study of the structure of Langmuir waves driven by a high-power laser pulse in a cluster plasma including experimental observation as well as simulations has been presented. The measurements from our oblique-angle frequency-domain holography diagnostic and simulations show that the wavefronts curve backwards, in contrast to the behavior observed in uniform plasma. Furthermore, these show that the wakefield wavelength behind the laser pulse is larger than in a uniform plasma but converges toward the uniform plasma value. This tunability of wavelengths using clusters has potential applications in overcoming the dephasing length in future laser-generated wakefield accelerators.

\section{ACKNOWLEDGMENTS}

The authors would like to acknowledge Christopher Arran for useful discussions. This work has been funded by EPSRC Grants No. EP/L000237/1 and No. EP/R029148/1, and by STFC Grants No. ST/M007375/1 and No. ST/ P002048/1. This research was also supported by the European Research Council (InPairs ERC-2015-AdG 
Grant No. 695088). The authors would like to thank the UCLA/IST Osiris consortium. We gratefully acknowledge all of the staff of the Central Laser Facility and the Scientific Computing Department at STFC Rutherford Appleton Laboratory. This work used the ARCHER UK National Supercomputing Service (http://www.archer.ac .uk) and STFCs SCARF cluster. One of the authors (K. G.) would like to thank the Rutherford International Fellowship Programme.

[1] L. Tonks and I. Langmuir, Oscillations in ionized gases, Phys. Rev. 33, 195 (1929).

[2] L. D. Landau, On the vibrations of the electronic plasma, Zh. Eksp. Teor. Fiz. 16, 574 (1946) [J. Phys.(USSR) 10, 25 (1946)].

[3] A. Bergmann and P. Mulser, Breaking of resonantly excited electron plasma waves, Phys. Rev. E 47, 3585 (1993).

[4] V. E. Zakharov, Collapse of langmuir waves, Sov. J. Exp. Theor. Phys 35, 908 (1972).

[5] J. M. Dawson, Nonlinear electron oscillations in a cold plasma, Phys. Rev. 113, 383 (1959).

[6] L. Spitzer, Physics of Fully Ionized Gases (Interscience Publishers, New York, 1956).

[7] C. Joshi, The development of laser- and beam-driven plasma accelerators as an experimental field, Phys. Plasmas 14, 055501 (2007).

[8] C. Joshi, E. Adli, W. An, C.E. Clayton, S. Corde, S. Gessner, M. J. Hogan, M. Litos, W. Lu, K. A. Marsh, W. B. Mori, N. Vafaei-Najafabadi, B. Oshea, X. Xu, G. White, and V. Yakimenko, Plasma wakefield acceleration experiments at FACET II, Plasma Phys. Controlled Fusion 60, 034001 (2018).

[9] C. Joshi and V. Malka, Focus on laser- and beam-driven plasma accelerators, New J. Phys. 12, 045003 (2010).

[10] R. Assmann et al. (AWAKE Collaboration), Proton-driven plasma wakefield acceleration: a path to the future of highenergy particle physics, Plasma Phys. Controlled Fusion 56, 084013 (2014).

[11] E. Gschwendtner et al., AWAKE, the advanced proton driven plasma wakefield acceleration experiment at CERN, Nucl. Instrum. Methods Phys. Res., Sect. A 829, 76 (2016).

[12] A. Caldwell et al., Path to AWAKE: Evolution of the concept, Nucl. Instrum. Methods Phys. Res., Sect. A 829, 3 (2016).

[13] I. Blumenfeld, C. E. Clayton, F.-J. Decker, M. J. Hogan, C. Huang, R. Ischebeck, R. Iverson, C. Joshi, T. Katsouleas, N. Kirby, W. Lu, K. A. Marsh, W. B. Mori, P. Muggli, E. Oz, R.H. Siemann, D. Walz, and M. Zhou, Energy doubling of $42 \mathrm{GeV}$ electrons in a metre-scale plasma wakefield accelerator, Nature (London) 445, 741 (2007).

[14] T. Tajima and J. M. Dawson, Laser Electron Accelerator, Phys. Rev. Lett. 43, 267 (1979).

[15] E. Esarey, C. B. Schroeder, and W. P. Leemans, Physics of laser-driven plasma-based electron accelerators, Rev. Mod. Phys. 81, 1229 (2009).
[16] D. Umstadter, Review of physics and applications of relativistic plasmas driven by ultra-intense lasers, Phys. Plasmas 8, 1774 (2001).

[17] A. Pukhov and J. Meyer-ter Vehn, Laser wake field acceleration: The highly non-linear broken-wave regime, Appl. Phys. B 74, 355 (2002).

[18] S.P.D. Mangles, C.D. Murphy, Z. Najmudin, A. G. R. Thomas, J.L. Collier, A.E. Dangor, E. J. Divall, P.S. Foster, J. G. Gallacher, C. J. Hooker, D. A. Jaroszynski, A. J. Langley, W. B. Mori, P. A. Norreys, F. S. Tsung, R. Viskup, B. R. Walton, and K. Krushelnick, Monoenergetic beams of relativistic electrons from intense laser-plasma interactions, Nature (London) 431, 535 (2004).

[19] C. G. R. Geddes, C. Toth, J. van Tilborg, E. Esarey, C. B. Schroeder, D. Bruhwiler, C. Nieter, J. Cary, and W. P. Leemans, High-quality electron beams from a laser wakefield accelerator using plasma-channel guiding, Nature (London) 431, 538 EP (2004).

[20] J. Faure, Y. Glinec, A. Pukhov, S. Kiselev, S. Gordienko, E. Lefebvre, J.-P. Rousseau, F. Burgy, and V. Malka, A laserplasma accelerator producing monoenergetic electron beams, Nature (London) 431, 541 (2004).

[21] W. P. Leemans, B. Nagler, A. J. Gonsalves, C. Tóth, K. Nakamura, C. G. R. Geddes, E. Esarey, C. B. Schroeder, and S. M. Hooker, GeV electron beams from a centimetrescale accelerator, Nat. Phys. 2, 696 (2006).

[22] O. Lundh, J. Lim, C. Rechatin, L. Ammoura, A. BenIsmaïl, X. Davoine, G. Gallot, J.-P. Goddet, E. Lefebvre, V. Malka, and J. Faure, Few femtosecond, few kiloampere electron bunch produced by a laser-plasma accelerator, Nat. Phys. 7, 219 (2011).

[23] M. Z. Mo, A. Ali, S. Fourmaux, P. Lassonde, J. C. Kieffer, and R. Fedosejevs, Quasimonoenergetic electron beams from laser wakefield acceleration in pure nitrogen, Appl. Phys. Lett. 100, 074101 (2012).

[24] X. Wang et al., Quasi-monoenergetic laser-plasma acceleration of electrons to $2 \mathrm{GeV}$, Nat. Commun. 4, 1988 (2013).

[25] H. T. Kim, K. H. Pae, H. J. Cha, I. J. Kim, T. J. Yu, J. H. Sung, S. K. Lee, T. M. Jeong, and J. Lee, Enhancement of Electron Energy to the Multi-GeV Regime by a Dual-Stage Laser-Wakefield Accelerator Pumped by Petawatt Laser Pulses, Phys. Rev. Lett. 111, 165002 (2013).

[26] W. P. Leemans, A. J. Gonsalves, H.-S. Mao, K. Nakamura, C. Benedetti, C. B. Schroeder, C. Tóth, J. Daniels, D. E. Mittelberger, S. S. Bulanov, J.-L. Vay, C. G. R. Geddes, and E. Esarey, Multi-GeV Electron Beams from CapillaryDischarge-Guided Subpetawatt Laser Pulses in the SelfTrapping Regime, Phys. Rev. Lett. 113, 245002 (2014).

[27] A. J. Gonsalves, K. Nakamura, J. Daniels, C. Benedetti, C. Pieronek, T. C. H. de Raadt, S. Steinke, J. H. Bin, S. S. Bulanov, J. van Tilborg, C. G. R. Geddes, C. B. Schroeder, C. Tóth, E. Esarey, K. Swanson, L. Fan-Chiang, G. Bagdasarov, N. Bobrova, V. Gasilov, G. Korn, P. Sasorov, and W. P. Leemans, Petawatt Laser Guiding and Electron Beam Acceleration to $8 \mathrm{GeV}$ in a Laser-Heated Capillary Discharge Waveguide, Phys. Rev. Lett. 122, 084801 (2019).

[28] V. Malka, J. Faure, Y. A. Gauduel, E. Lefebvre, A. Rousse, and K. T. Phuoc, Principles and applications of compact laser-plasma accelerators, Nat. Phys 4, 447 (2008). 
[29] P. A. Norreys, Laser-driven particle acceleration, Nat. Photonics 3, 423 (2009).

[30] S. M. Hooker, Developments in laser-driven plasma accelerators, Nat. Photonics 7, 775 (2013).

[31] Y. Fukuda, Y. Akahane, M. Aoyama, Y. Hayashi, T. Homma, N. Inoue, M. Kando, S. Kanazawa, H. Kiriyama, S. Kondo, H. Kotaki, S. Masuda, M. Mori, A. Yamazaki, K. Yamakawa, E. Echkina, I. Inovenkov, J. Koga, and S. Bulanov, Ultrarelativistic electron generation during the intense, ultrashort laser pulse interaction with clusters, Phys. Lett. A 363, 130 (2007).

[32] L. Zhang, L.-M. Chen, W.-M. Wang, W.-C. Yan, D.-W. Yuan, J.-Y. Mao, Z.-H. Wang, C. Liu, Z.-W. Shen, A. Faenov, T. Pikuz, D.-Z. Li, Y.-T. Li, Q.-L. Dong, X. Lu, J.-L. Ma, Z.-Y. Wei, Z.-M. Sheng, and J. Zhang, Electron acceleration via high contrast laser interacting with submicron clusters, Appl. Phys. Lett. 100, 014104 (2012).

[33] M. Mirzaie, N. A. M. Hafz, S. Li, K. Gao, G. Li, Q. Ul-Ain, and J. Zhang, Laser acceleration in argon clusters and gas media, Plasma Phys. Controlled Fusion 58, 034014 (2016).

[34] J. Wood, Betatron radiation from laser wakefield accelerators and its applications, Ph.D. thesis, Imperial College London, 2017.

[35] T. Tajima, Y. Kishimoto, and M. C. Downer, Optical properties of cluster plasma, Phys. Plasmas 6, 3759 (1999).

[36] T. Ditmire, T. Donnelly, A. M. Rubenchik, R. W. Falcone, and M. D. Perry, Interaction of intense laser pulses with atomic clusters, Phys. Rev. A 53, 3379 (1996).

[37] H. M. Milchberg, S. J. McNaught, and E. Parra, Plasma hydrodynamics of the intense laser-cluster interaction, Phys. Rev. E 64, 056402 (2001).

[38] A. Gupta, T. M. Antonsen, and H. M. Milchberg, Propagation of intense short laser pulses in a gas of atomic clusters, Phys. Rev. E 70, 046410 (2004).

[39] B. N. Breizman, A. V. Arefiev, and M. V. Fomytskyi, Nonlinear physics of laser-irradiated microclusters, Phys. Plasmas 12, 056706 (2005).

[40] T. M. Antonsen, T. Taguchi, A. Gupta, J. Palastro, and H. M. Milchberg, Resonant heating of a cluster plasma by intense laser light, Phys. Plasmas 12, 056703 (2005).

[41] D. R. Symes, M. Hohenberger, A. Henig, and T. Ditmire, Anisotropic Explosions of Hydrogen Clusters under Intense Femtosecond Laser Irradiation, Phys. Rev. Lett. 98, 123401 (2007).

[42] T. Fennel, K.-H. Meiwes-Broer, J. Tiggesbäumker, P.-G. Reinhard, P. M. Dinh, and E. Suraud, Laser-driven nonlinear cluster dynamics, Rev. Mod. Phys. 82, 1793 (2010).

[43] M. F. Kasim, J. Holloway, L. Ceurvorst, M. C. Levy, N. Ratan, J. Sadler, R. Bingham, P. N. Burrows, R. Trines, M. Wing, and P. Norreys, Quantitative single shot and spatially resolved plasma wakefield diagnostics, Phys. Rev. Accel. Beams 18, 081302 (2015).

[44] C. J. Hooker, J. L. Collier, O. Chekhlov, R. Clarke, E. Divall, K. Ertel, B. Fell, P. Foster, S. Hancock, A. Langley, D. Neely, J. Smith, and B. Wyborn, The Astra Gemini project - A dual-beam petawatt Ti:Sapphire laser system, J. Phys. IV France 133, 673 (2006).

[45] O.F. Hagena, Cluster ion sources (invited), Rev. Sci. Instrum. 63, 2374 (1992).

[46] R. A. Smith, T. Ditmire, and J. W. G. Tisch, Characterization of a cryogenically cooled high-pressure gas jet for laser/cluster interaction experiments, Rev. Sci. Instrum. 69, 3798 (1998).

[47] Z. Li, H.-E. Tsai, X. Zhang, C.-H. Pai, Y.-Y. Chang, R. Zgadzaj, X. Wang, V. Khudik, G. Shvets, and M.C. Downer, Single-Shot Visualization of Evolving Laser Wakefields Using an All-Optical Streak Camera, Phys. Rev. Lett. 113, 085001 (2014).

[48] K. Y. Kim, I. Alexeev, and H. M. Milchberg, Single-shot supercontinuum spectral interferometry, Appl. Phys. Lett. 81, 4124 (2002).

[49] N. H. Matlis, S. Reed, S. S. Bulanov, V. Chvykov, G. Kalintchenko, T. Matsuoka, P. Rousseau, V. Yanovsky, A. Maksimchuk, S. Kalmykov, G. Shvets, and M. C. Downer, Snapshots of laser wakefields, Nat. Phys. 2, 749 EP (2006).

[50] E. Esarey, A. Ting, P. Sprangle, D. Umstadter, and X. Liu, Nonlinear analysis of relativistic harmonic generation by intense lasers in plasmas, IEEE Trans. Plasma Sci. 21, 95 (1993).

[51] D. Teychenné, G. Bonnaud, and J.-L. Bobin, Wave-breaking limit to the wake-field effect in an underdense plasma, Phys. Rev. E 48, R3248 (1993).

[52] P. Sprangle, E. Esarey, and A. Ting, Nonlinear interaction of intense laser pulses in plasmas, Phys. Rev. A 41, 4463 (1990).

[53] P. Sprangle, E. Esarey, and A. Ting, Nonlinear Theory of Intense Laser-Plasma Interactions, Phys. Rev. Lett. 64, 2011 (1990).

[54] C. Shu, High order weighted essentially nonoscillatory schemes for convection dominated problems, SIAM Rev. 51, 82 (2009).

[55] R. A. Fonseca, L. O. Silva, F. S. Tsung, V. K. Decyk, W. Lu, C. Ren, W. B. Mori, S. Deng, S. Lee, T. Katsouleas, and J. C. Adam, in Computational Science-ICCS 2002, edited by P. M. A. Sloot, A. G. Hoekstra, C. J. K. Tan, and J. J. Dongarra (Springer, Berlin, Heidelberg, 2002), pp. 342-351.

[56] H. Lu, G. Chen, G. Ni, R. Li, and Z. Xu, Impact of gas backing pressure and geometry of conical nozzle on the formation of methane clusters in supersonic jets, J. Phys. Chem. A 114, 2 (2010).

[57] A. Murakami, J. Miyazawa, H. Tsuchiya, T. Murase, N. Ashikawa, T. Morisaki, R. Sakamoto, and H. Yamada, Characteristics of hydrogen supersonic cluster beam generated by a Laval nozzle, J. Plasma Fusion Res. SERIES 9, 79 (2010).

[58] E. Lindman, "Free-space" boundary conditions for the time dependent wave equation, J. Comput. Phys. 18, 66 (1975).

[59] P. K. Tiwari and V. K. Tripathi, Stimulated Raman scattering of a laser in a plasma with clusters, Phys. Plasmas 11, 1674 (2004). 